\title{
Clinical Benefits of Emotional Freedom Techniques on Food Cravings at 12-Months Follow-Up: A Randomized Controlled Trial
}

\author{
Peta Stapleton, Bond University, Australia \\ Teri Sheldon, The Lakeside Rooms, Australia \\ Brett Porter, The Lakeside Rooms, Australia
}

\begin{abstract}
This randomized, single-blind, crossover trial tested whether participants who used Emotional Freedom Techniques (EFT) maintained reduced food cravings after 12-months and updates previously reported 6 -month findings. Ninety-six overweight/obese adults were allocated to a 4-week EFT treatment or waitlist condition. Degree of food craving, perceived power of food, restraint capabilities, and psychological symptoms were assessed pre- and posttest and at 12-month follow-up for collapsed groups. Significant improvements occurred in weight, body
\end{abstract}

mass index, food cravings, subjective power of food, craving restraint, and psychological coping for EFT participants from pretest to 12 months $(p<.05)$. It appears EFT can result in participants maintaining reduced cravings over time and affect weight and BMI in overweight and obese individuals.

Keywords: EFT, Emotional Freedom Techniques, cravings, psychological symptoms, food, obesity.
Peta Stapleton, $\mathrm{PhD}$, has 19 years experience as a registered clinical psychologist in the State of Queensland, Australia and is Assistant Professor in the Faculty of Humanities and Social Sciences at Bond University, Queensland, Australia. Terri Sheldon, BA Hons, is a registered psychologist in the State of Queensland, Australia, and certified practitioner of Emotional Freedom Techniques (EFT), The Lakeside Rooms, Gold Coast, Australia. Brett Porter is a therapist and certified practitioner of EFT, The Lakeside Rooms, Gold Coast, Australia. Acknowledgments: The authors thank Wedderburn for their generous donation of the Tanita Scales for the duration of the trial, the Lakeside Rooms for their clinical support and access to treatment facilities, and the Association for Comprehensive Energy Psychology for seed funding. Correspondence concerning this article should be sent to Peta Stapleton, Faculty of Humanities and Social Sciences, University Drive, Bond University, Robina, Gold Coast 4229, Queensland Australia or pstaplet@bond.edu.au. Disclosures: The authors declare no conflicts of interest.

$\mathrm{T}$ he need to prevent future global obesity has highlighted the necessity of early identification of elevated body mass indexes (BMI), medical risks, and unhealthy eating and physical activity habits. A recent systematic review of the direct costs of obesity (Withrow \& Alter, 2010) revealed that, depending on the country, obesity accounts for between $0.7 \%$ and $2.8 \%$ of total healthcare expenditures. Obesity is a chronic disease, and the need for its effective management strategies is paramount.

One area of interest in the obesity epidemic is the role food cravings play in maintaining excessive eating patterns (Weingarten \& Elston, 1990). It has been suggested that food cravings frequently lead to consumption of the craved food (Hill \& Heaton-Brown, 1994) are positively correlated with BMI (Delahanty, Meigs, Hayden, Williamson, \& Nathan, 2002; Franken \& Muris, 2005), and obese adults have reported preferences for high fat foods (Drewnowski, Brunzell, Sande, Iverius, \& Greenwood, 1985). Even in nonclinical samples, food craving has been found to be related to body weight, suggesting the role of craving in food consumption (Franken \& Muris, 2005).

We know weight loss for obese people results in health benefits that include reductions in cardiovascular risk factors and improvement in psychological coping (Garrow \& Summerbell, 2000). However, current weight loss/body shape change programs are based largely on principles of balancing caloric intake with expenditure: eating less and exercising more. Few studies have focused 
on strategies to reduce food cravings. Approaches such as thought suppression (ignoring craving related thoughts) or cue-response response-prevention (not allowing an individual to eat a food after it has been presented) have not been overly successful (Kemps \& Tiggemann, 2010). New and distinctive craving reduction strategies are necessary, and if applied within obese populations, it is crucial to ascertain their long-term effectiveness and impact upon weight and BMI. Meta-analysis of weight loss programs with the addition of psychological techniques has confirmed that people who are overweight or obese also benefit from psychological interventions to enhance weight reduction, particularly behavioral and cognitivebehavioral strategies (Shaw, O'Rourke, Del Mar, $\&$ Kenardy, 2005), and these may be useful in targeting food cravings.

\section{Psychological Intervention for Food Cravings}

The addition of meridian-based procedures using acupressure points (e.g., Emotional Freedom Techniques; EFT) has shown merit when applied to food cravings. A meridian system is commonly referred to as the body's "energy field" or "electrical system" (Feinstein, 2008), and procedures that target the meridian system are often titled energy psychology techniques. Techniques such as EFT combine cognitive strategies with somatic procedures adapted from acupuncture and related systems for altering the cognitive, behavioral, and neurochemical foundations of psychological problems. Several thousand clinical reports exist that support EFT, and a growing catalog of clinical trials has supported its efficacy with a range of conditions, for example, posttraumatic stress disorder (Church \& Brooks, 2010; Karatzias et al., 2011; Stein \& Brooks, 2011), specific phobias (Salas, Brooks, \& Rowe, 2011; Wells, Polglase, Andrews, Carrington, \& Baker, 2003), and weight loss maintenance (Elder et al., 2007).

In Stapleton, Sheldon, Porter, and Whitty (2011), three of the current authors demonstrated EFT to be associated with a significantly greater improvement in food cravings, the subjective power of food and craving restraint when compared to a waitlist, from pretest to immediately posttest $(p<.05)$. Across collapsed groups, which were combined for analysis, an improvement in food cravings and the subjective power of food after treatment was maintained at 6-months, and a delayed effect was seen for food restraint (specific data are presented in more detail in the Results section). The present study is an extension of this trial and reports the 12-month follow-up results. It was expected that the treatment gains made at 6-months would be maintained in the EFT group at 12-months.

\section{The Present Study}

The authors' recent investigation of EFT for food cravings involved 96 overweight or obese adults who were randomized into an active treatment group who received group-delivered EFT or a matched waitlist (WL) who received no treatment for the same period of time as the treatment offered. The treatment group received 2 hour of EFT for 4 weeks, and at the end of the waiting period (4 weeks), the WL were offered the same treatment. All WL participants began and completed the treatment exactly 4 weeks from pretest to when they began the treatment; there were no withdrawals. Food craving, perceived power of food, restraint capabilities, and psychological symptoms, as well as weight measurements were assessed pretest, posttest, and 6 and 12 months after the treatment program.

A statistician unconnected to the study and blind to its aims who was associated with the lead author's institution conducted randomization using a computerized random-number generator. All participants chose one food to address in their treatment group from the following list of groups: salty foods (e.g., chips, crisps, salted nuts), sweet carbohydrate foods (e.g., cakes, cookies, soft/soda drinks), carbohydrate foods that are neither sweet nor salty, such as white refined foods (e.g., bread, rice, pasta), and caffeinated products.

\section{Method}

We obtained ethics approval from the Griffith University with which the lead author was then affiliated. Male and female participants were recruited through community announcements in print advertisements, radio, and television. Participants needed to (a) be over 18 years old and under 60 years and not be suffering from any severe psychological impairment, (b) not currently be receiving treatment (psychological or medical) for their food cravings, (c) agree to be contacted 
for follow-up testing, (d) have a BMI greater than 25, and (e) have food cravings of a certain severity (defined below). Active diagnoses from the Symptom Assessment-45 questionnaire (SA45; Strategic Advantages, 2000) and participants taking psychotropic medication that can suppress feelings, cravings, and sensitivity were excluded. Individuals with diabetes (Type I and II) and hypoglycaemia and women who were pregnant were excluded due to any physiological aetiology (effects related) to food cravings. Any individuals diagnosed with anorexia nervosa were also excluded. Potential participants who did not meet criteria were offered a referral list of professionals to access individually. A total of 158 participants were screened, 38 were excluded for not meeting eligibility, and 120 participants were offered a place in the trial; 24 withdrew for personal reasons, and 96 were then randomized into the treatment or WL condition. All 96 participants completed an informed consent process. Figure 1 describes the flow of participants through the trial.

A telephone screening procedure was used to determine eligibility, and potential participants completed the Food Craving Inventory (FCI; White, Whisenhunht, Williamson, Greenway, \& Netemeyer, 2002). The SA-45, a short form of the Symptom Checklist (SCL-90), was also used in the screening process to assess symptomatology across nine psychiatric domains. In the screening process, and due to it being delivered via the telephone, participants were asked to self-report their weight and height in order to ascertain their BMI. A self-report questionnaire package was issued to eligible participants, and they were asked to complete a demographic questionnaire, the FCI, the Power of Food Scale (POF; Lowe et al., 2009), and the SA-45. Three aspects of eating behavior (cognitive restraint, uncontrolled eating, and emotional eating) were also measured using the Revised Restraint Scale (Herman \& Polivy, 1980). The heights and weights of eligible participants were measured in person by the chief investigators at the pre- and posttreatment points, but they were again self-reported by participants at the 6- and 12-month time points. See Stapleton et al. (2011) for further details.

\section{Self-Report Measures}

The FCI (White et al., 2002) is a reliable and valid measure for the assessment of cravings for
37 specific types of foods over the past month. It has 28 items from four scales: High Fats, Sweets, Carbohydrates/Starches, and Fast Food Fats (nine items are not scored). All items are rated on a 5-point Likert scale, and the 28 scores from the four scales comprise the higher order construct of "food craving," or the FCI Total score. Higher numbers for each of the subscales reflect greater cravings for that food type, with the highest score being 185 .

The POF was originally developed to assess the psychological impact of living in environments in which highly appetizing food was readily available, and the authors suggest that learning to have control over one's own eating environment is critical for long-term weight loss and maintenance. The POF is a 21-item scale, with each item rated on a 5-point Likert scale, and it specifically measures the psychological influence of available food. It has been found to be internally consistent and temporally stable over a 4-month period (Lowe et al., 2009).

In the Revised Restraint Scale (Herman \& Polivy, 1980) each item is scored on a scale of 0 to 4 , and the items are summed for a total score that ranges from 0 to 40 . High scores indicate chronic dieting, where the individual is constantly cycling on and off a diet, typically without any substantial weight loss. The scale is valid in normal-weight samples (Heatherton, Herman, Polivy, King, \& McGree, 1988) and has high test-retest reliability and sound internal consistency (Ruderman \& Besbeas, 1992). Dietary restraint appears to play a causal role in the development of eating disorders, obesity, and loss of control over intake. Food cravings may trigger these eating behaviors.

The SA-45 was used to assess symptomatology across nine psychiatric domains and as measures of the outcome of the EFT intervention. It is a short form of the SCL-90 and uses the proven items and structure of the SCL-90 Revised to create a brief measure of psychiatric symptomatology. The SA-45 uses a 5-point Likert scale and measures Anxiety, Depression, Hostility, Interpersonal Sensitivity, Obsessive-Compulsivity, Paranoid Ideation, Phobic Anxiety, Psychoticism, and Somatization. The SA-45 also features a Positive Symptom Index (PST), which indicates the total number of symptoms reported to be present (i.e., item yielding a response other than "not at all"), and a Global Severity Index (GSI), which represents the total of the item response values for all 


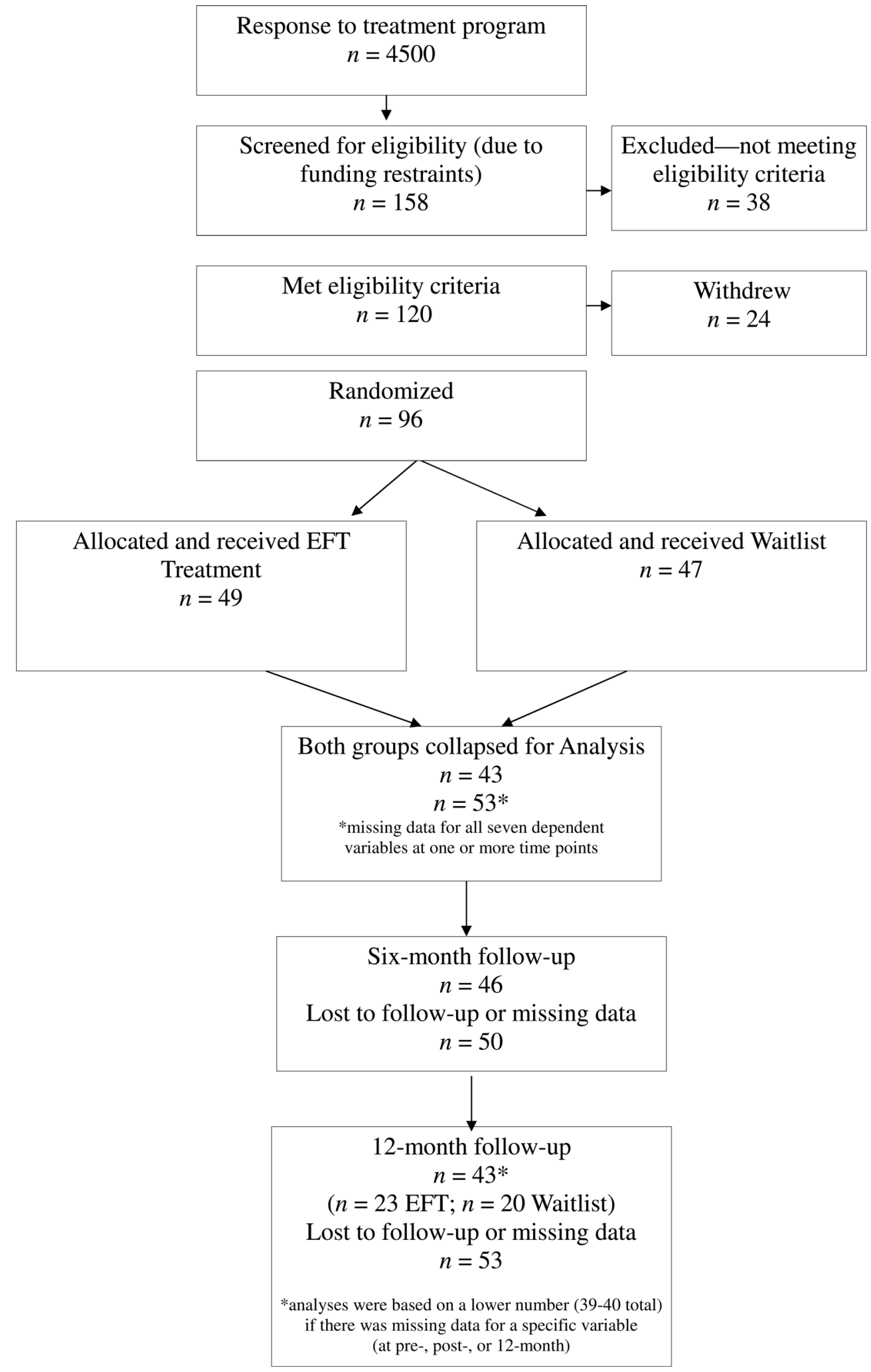

Figure 1 CONSORT Flow of Participants Through Trial 
items on the SA-45. This provides a more meaningful overview of the level of symptomatology. As a brief form of the SCL-90, the SA-45 has demonstrated sound psychometric properties (normative data, reliability, and validity; Sandín, Valiente, Chorot, Santed, \& Lostao, 2008). It is often used as a screening tool and to measure outcomes.

\section{Treatment Condition}

The EFT treatment was conducted by a certified EFT practitioner. All the treatment conditions were delivered in groups of 15 at a local therapy clinic. The EFT intervention consisted of four sessions ( 2 hour duration each) with homework and was based on standardized treatment protocols (Craig \& Fowlie, 1995). Full instructions and safeguards are described in Flint, Lammers, and Mitnick (2005). In the present study, acupressure points one to seven (eyebrow, side of eye, under eye, under nose, chin, collarbone, under arm) and the top of the head (eight) were used, and the ninegamut sequence was omitted due to Craig's (2008) suggestion that it is no longer necessary for treatment outcomes. The specific session topics were (a) psychoeducation about EFT and how it works; (b) the nature of food cravings and how they can be addressed with EFT; (c) feelings and food, and (d) relapse prevention, using EFT for stress and relaxation and goal setting. These sessions formed the basis of the EFT and Food Craving manual, which was designed for this study by the authors and the EFT practitioner. The homework tasks were specifically to practice the techniques taught during the week in between treatment sessions.

A treatment fidelity plan was formed prior to the trial commencing. The chief investigators and the EFT practitioner developed and reviewed the treatment manual to ensure that the active ingredients of the EFT intervention were fully operationalized. Fidelity was not monitored via video in order to protect patient privacy. Instead, intervention checklists were used, and participants completed social validity scales each week to evaluate the program and the EFT practitioner. On a 7-point Likert scale where 0 represented not at all, and 6 equalled very much, $91 \%$ of participants indicated EFT addressed their concerns very well, and $89 \%$ found the EFT program "very much" met their goals for reducing food cravings. On a 5-point Likert scale, where 1 equalled not at all and 5 equalled very much, $95 \%$ found the facilitator to be very approachable.

\section{Data Analysis}

Data were analyzed using SPSS-17 (SPSS for Windows, 2008). For all analyses, we excluded individuals with missing data at any time point on an analysis-by-analysis basis. To test whether the effect of EFT was sustained at 12-months, we used seven separate repeated measure analyses of variance (ANOVAs) to measure the effect of three levels of the repeated measure variable of time (pretreatment, posttreatment, 12 months). The seven outcome variables were weight, BMI, FCI total score, POF total score, Revised Restraint total score, SA45 GSI score, and SA45 PST score. To maximize the sample size, we collapsed the two groups EFT and WL into one group (the WL received active treatment after completion of the test period). Paired comparisons between time points were undertaken using post hoc tests. The Bonferroni correction was applied for multiple comparisons.

Analysis was based on a total of 96 participants (EFT, $n=49$; WL, $n=47$ ). Twelve month data were available for 43 participants (23 in EFT and 20 in WL groups); however, analyses were based on a lower number (39-40 total) if there were missing data for a specific variable (at pretreatment, posttreatment, or 12 month follow-up). Although previously published, the 6-month data are included (Stapleton et al., 2011). The observation was excluded on an analysis-by-analysis basis. Reasons for attrition included residential move and changes in electronic addresses (email) and telephone contact details, which prevented follow-up questionnaires being delivered. For all participants, contact was attempted at each point a total of three times. No adverse events were reported to any of the researchers by any participant.

\section{Results}

Baseline characteristics of participants at recruitment and initial craving concerns (chocolate was the most commonly chosen food craving) are presented in detail in Stapleton et al. (2011). The majority of participants were women (89\%), were over 40 years of age (68\%), married $(53.1 \%)$, did not live alone $(87 \%)$, had undertaken some form of education since high school $(60 \%)$, were employed (79\%), and had a household income exceeding $\$ 50,000(65 \%)$. There were no significant differences between the EFT and WL conditions in baseline sociodemographic characteristics $(p>$ 
$.05)$. There were also no significant differences in the recruitment weight (mean $90.52 \pm 19.11 \mathrm{~kg}$ or $199.1 \pm 42 \mathrm{lbs}$ ), BMI (mean $32.78 \pm 6.03$ ), and selfreport measures between the EFT and WL groups $(p>.05)$.

\section{Review of 6-Month Follow-Up}

Although these data were presented in Stapleton et al. (2011), they are offered here as a summary.

At the 6-month time point, repeated measure ANOVA analyses indicated (within subject) a significant interaction over time for FCI total scores and the POF scale, indicating participants received a significant change over time. The SA45GSI and SA45PST scales were also significantly different from pre- to posttreatment points, indicating participants were experiencing a reduction in psychological distress.

Weight and BMI. There were no significant mean differences between the three time points for weight (pretreatment vs. posttreatment mean difference. $-0.57, p=.096 ; 6$ months vs. pretreatment mean difference, $-2.81, p=.475 ; 6$ months vs. posttreatment mean difference, $-2.24, p=$ .767) and BMI (pretreatment vs. posttreatment mean difference, $-0.20, p=.397 ; 6$ months vs. pretreatment mean difference, $-0.95, p=.653 ; 6$ months vs. posttreatment mean difference, -0.76 , $p=.997)$.

FCI total. There was a significant reduction from pretreatment to immediately posttreatment for FCI total scores (mean difference, $-16.00, p<.001$ ), and this was maintained at 6 months (mean difference, $-10.17, p<.05)$.

POF. There was a significant reduction from pretreatment to immediately posttreatment for the POF scale (mean difference, $-23.20, p<.001$ ), and this reduction was maintained at the 6 month follow-up (mean difference, $-27.24, p<.001$ ).

Total restraint score. The mean difference for the restraint scale was not significant immediately posttreatment (mean difference, $-1.71, p<$ .05 ) but reached significance at the 6-month point (mean difference, -3.46 from pretreatment, $p<$ $.01)$. There was a further reduction in the restraint score (mean difference, $-1.76, p=.028$ ) between posttreatment and 6-month follow-up, indicating participants were approaching a more "normal" internal regulation of food intake.

SA-45. For the psychological distress scores, there was a significant reduction in distress from pre- to posttreatment (SA45GSI mean difference, -3.44 , $p<.05$; SA45PST mean difference, $-3.56, p<$ .01 ), indicating participants were experiencing a reduction in psychological distress immediately following treatment. Both the SA-45 GSI and PST scores were significantly reduced at 6 months compared to pretreatment $(p<.05)$.

\section{The Effect of EFT Over 12 Months}

Table 1 shows the means and standard deviations at pretreatment, posttreatment, and 6- and 12-months follow-up for the collapsed groups. Table 2 presents selected post hoc paired comparisons, with Bonferroni adjustment, for repeated measures ANOVAs (based on $n=39$ or 40 ).

There was a significant difference pretreatment to posttreatment to 12-month follow-up in all measures: participants' weight, BMI, FCI total, POF total, Revised Restraint, SA45GSI, and SA45PST $(p<.05$; Table 1$)$, indicating participants demonstrated a significant change across these measures over time.

Table 2 presents post hoc comparisons across the three time points. There were significant reductions from pretreatment to 12-months for participants' weight (mean difference, $-5.05 \mathrm{~kg}$ or $11.1 \mathrm{lb}, p<.05$ ), BMI (mean difference, $-2.28, p<$ .05 ), FCI total scores (mean difference, $-13.40, p$ $<.001$ ), POF total score (mean difference, -25.33 , $p<.001$ ), Restraint (mean difference, $-3.08, p$ $<.001$ ), and SA45GSI (mean difference, 5.47, $p<.05)$. There was no significant difference for SA45PST pretreatment to 12 months (mean difference, $-4.62, p<.05)$.

From posttreatment versus 12 months, there were significant reductions for participants' weight (mean difference, $-4.65 \mathrm{~kg} / 10.2 \mathrm{lb}, \mathrm{p}<0.05$ ), and BMI (mean difference, $-2.13, p<.05$ ). There was no significant difference for FCI total scores, POF total score, Restraint, SA45GSI, and SA45PST scores from posttreatment to 12-months followup. However, it is worth noting that the significant reductions in these measures achieved from preto posttreatment were maintained at the 6-month point (with the exception of Restraint, which reached significance at 6 months). 
Table 1 Means, Standard Deviations at Pre-, Post-, 6-Months, and 12-Months for Collapsed Groups

\begin{tabular}{|c|c|c|c|c|c|c|c|c|c|c|c|c|}
\hline \multirow[b]{2}{*}{ Variable } & \multirow[b]{2}{*}{$n$} & \multicolumn{2}{|c|}{ Pretreatment } & \multicolumn{2}{|c|}{ Posttreatment } & \multicolumn{2}{|c|}{6 month } & \multicolumn{2}{|c|}{12 month } & \multirow[b]{2}{*}{$d f$} & \multirow[b]{2}{*}{$F$} & \multirow[b]{2}{*}{$P$} \\
\hline & & $M$ & $S D$ & $M$ & $S D$ & $M$ & $S D$ & $M$ & $S D$ & & & \\
\hline Weight & 40 & $\begin{array}{l}92.25 \mathrm{~kg} / \\
203 \mathrm{lbs}\end{array}$ & 18.73 & $\begin{array}{l}91.85 \mathrm{~kg} / \\
202.1 \mathrm{lbs}\end{array}$ & 18 & $\begin{array}{l}90.16 \mathrm{~kg} / \\
198.4 \mathrm{lbs}\end{array}$ & 17.71 & $\begin{array}{l}87.20 \mathrm{~kg} / \\
191.8 \mathrm{lbs}\end{array}$ & & 1.04 & 9.81 & .003 \\
\hline BMI & 40 & 33.00 & 6.60 & 32.85 & 6.83 & 32.34 & 6.46 & 30.73 & 6.56 & $1.04,400.57$ & 13.73 & .001 \\
\hline FCI Total & 40 & 61.87 & 16.39 & 47.93 & 12.58 & 53.38 & 18.58 & 48.47 & 13.80 & 2,78 & 24.58 & $<.001$ \\
\hline POF Total & 39 & 71.79 & 19.79 & 52.21 & 18.09 & 49.68 & 19.48 & 46.46 & 18.67 & 2,76 & 30.74 & $<.001$ \\
\hline $\begin{array}{l}\text { Restraint } \\
\text { Total }\end{array}$ & 39 & 32.59 & 4.88 & 31.41 & 4.73 & 30.46 & 5.84 & 29.51 & 4.83 & 2,76 & 8.78 & $<.001$ \\
\hline SA45GSI & 45 & 59.20 & 9.80 & 56.24 & 7.70 & 53.02 & 10.03 & 53.73 & 9.60 & 2,88 & 8.94 & $<.001$ \\
\hline SA45PST & 45 & 58.56 & 9.54 & 56.09 & 8.34 & 53.66 & 10.65 & 53.93 & 10.52 & 2,88 & 5.88 & .004 \\
\hline
\end{tabular}

Note . BMI = Body Mass Index, FCI = Food Craving Inventory, POF $=$ Power of Food Scale, Restraint $=$ Revised Restraint Scale, SA45GSI = Symptom Assessment Global Severity Index, SA45PST = Symptom Assessment Positive Symptom Index.

Table 2 Post Hoc Paired Comparisons, With Bonferroni Adjustment, for Repeated Measures Analyses of Variance

\begin{tabular}{|c|c|c|c|c|c|c|c|}
\hline \multirow[b]{2}{*}{ Variable } & \multirow[b]{2}{*}{$n$} & \multicolumn{2}{|l|}{$\begin{array}{l}\text { 12-month vs. } \\
\text { pretreatment }\end{array}$} & \multicolumn{2}{|c|}{$\begin{array}{l}\text { 12-month vs. } \\
\text { posttreatment }\end{array}$} & \multicolumn{2}{|c|}{$\begin{array}{c}\text { Post vs. } \\
\text { pretreatment }\end{array}$} \\
\hline & & Mean difference & $p$ & Mean difference & $p$ & Mean difference & $p$ \\
\hline Weight & 40 & $-5.05 \mathrm{~kg} / 11.1 \mathrm{lbs}$ & .006 & $-4.65 \mathrm{~kg} / 10.2 \mathrm{lbs}$ & $* 0.014$ & $-0.40 \mathrm{~kg} / 0.9 \mathrm{lbs}$ & .407 \\
\hline BMI & 40 & -2.28 & .001 & -2.13 & $* 0.003$ & -0.15 & .406 \\
\hline FCI Total & 40 & -13.40 & $<.001$ & 0.55 & 1.000 & -13.95 & $<.001$ \\
\hline POF Total & 39 & -25.33 & $<.001$ & -5.74 & 0.221 & -19.59 & $<.001$ \\
\hline Restraint Total & 39 & -3.08 & $<.001$ & -1.90 & 0.069 & -1.18 & .292 \\
\hline SA45GSI & 45 & -5.47 & .001 & -2.51 & 0.257 & -2.96 & .029 \\
\hline SA45PST & 45 & -4.62 & .013 & -2.16 & 0.334 & -2.47 & $.117^{\mathrm{a}}$ \\
\hline
\end{tabular}

Note . BMI = Body Mass Index, FCI = Food Craving Inventory, POF = Power of Food Scale, Restraint $=$ Revised Restraint Scale, SA45GSI = Symptom Assessment Global Severity Index, SA45PST = Symptom Assessment Positive Symptom Index.

${ }^{a}$ Cohen's $d$ was .67, which suggests a medium to large effect. The difference from post- to pretreatment. was inconclusive, as the power was low.

All the possible time point contrasts were also analyzed (e.g., pre- vs. posttreatment, pretreatment vs. 6- and 12-month follow-up, posttreatment vs. 6- and 12-month follow-up, and 6-month vs. 12-month follow-up; see Table 3). Because of missing data for some of the time periods, repeated measures ANOVA with post hoc comparisons were not used, instead individual paired-sample $t$ tests were chosen to maximise the sample size for each comparison. BMI at 12 months $(M=31.91$, $S D=7.79)$ was significantly lower than at pretreatment $(M=34.00, S D=7.57)$ and also significantly lower than posttreatment $(M=33.87$, $S D=7.69$ ). Compared to pretreatment, food crav- ing was decreased at 6-month $(M=52.63, S D$ $=17.46)$ and 12-month follow-up $(M=49.74$; $S D=14.85)$. Power over food at 6-month $(M=$ $50.58, S D=19.81)$ and 12-month follow-up $(M=$ $49.53, S D=20.13)$ was significantly lower than at pretreatment $(M=77.56, S D=18.74)$. Restraint at 6 months $(M=30.51, S D=5.84)$ was significantly improved over pretreatment $(M=33.60$, $S D=5.34)$ and when pretreatment was compared to 12-month follow-up $(M=29.57, S D=5.01)$. Furthermore, restraint at 12-month follow-up was significantly improved when compared to posttreatment $(M=31.76, S D=4.76)$. The SA45 global score was improved from pre treatment 
Table 3 Individual Paired Sample t Tests Across All Time Points, for Collapsed Groups

\begin{tabular}{|c|c|c|c|c|c|}
\hline Pair & $n$ & $M$ & $S D$ & $d f$ & $p$ \\
\hline BMI pre & 98 & 33.84 & 7.162 & 97 & .093 \\
\hline BMI post & 98 & 33.37 & 6.648 & & \\
\hline BMI pre & 48 & 33.92 & 7.022 & 47 & .124 \\
\hline BMI 6months & 48 & 32.85 & 7.182 & & \\
\hline BMI pre & 48 & 34.00 & 7.570 & 46 & $<.001$ \\
\hline BMI 12 months & 48 & 31.91 & 7.799 & & \\
\hline BMI post & 47 & 33.79 & 7.065 & 46 & .263 \\
\hline BMI 6 months & 47 & 33.00 & 7.187 & & \\
\hline BMI post & 45 & 33.87 & 7.695 & 44 & .002 \\
\hline BMI 12 months & 45 & 32.00 & 7.917 & & \\
\hline BMI 6 months & 28 & 32.82 & 7.503 & 27 & .055 \\
\hline BMI 12 months & 28 & 31.61 & 8.359 & & \\
\hline FCI pre & 99 & 63.99 & 15.049 & 98 & $<.001$ \\
\hline FCI post & 99 & 49.51 & 14.454 & & \\
\hline FCI pre & 49 & 64.86 & 15.764 & 48 & $<.001$ \\
\hline FCI 6months & 49 & 52.63 & 17.466 & & \\
\hline FCI pre & 46 & 64.11 & 17.323 & 45 & $<.001$ \\
\hline FCI 12 months & 46 & 49.74 & 14.853 & & \\
\hline FCI post & 49 & 54.73 & 19.503 & 48 & .704 \\
\hline FCI 6 months & 49 & 53.37 & 17.849 & & \\
\hline FCI post & 46 & 51.02 & 16.212 & 45 & .838 \\
\hline FCI 12 months & 46 & 5.61 & 16.542 & & \\
\hline FCI 6 months & 29 & 55.38 & 2.184 & 28 & .015 \\
\hline FCI 12 months & 29 & 49.34 & 17.034 & & \\
\hline POF pre & 98 & 73.88 & 19.373 & 97 & $<.001$ \\
\hline POF post & 98 & 55.23 & 19.057 & & \\
\hline POF pre & 48 & 77.56 & 18.739 & 47 & $<.001$ \\
\hline POF 6 months & 48 & 5.58 & 19.807 & & \\
\hline POF pre & 48 & 72.36 & 19.315 & 47 & $<.001$ \\
\hline POF 12 months & 48 & 49.53 & 2.129 & & \\
\hline POF post & 49 & 54.73 & 19.503 & 48 & .328 \\
\hline POF 6 months & 49 & 51.80 & 2.955 & & \\
\hline POF post & 46 & 53.67 & 18.808 & 45 & .328 \\
\hline POF 12 months & 46 & 49.93 & 21.500 & & \\
\hline POF 6 months & 29 & 52.76 & 22.299 & 28 & .974 \\
\hline POF 12 months & 29 & 52.86 & 21.198 & & \\
\hline Rest pre & 96 & 33.39 & 5.466 & 95 & .001 \\
\hline Rest post & 96 & 31.74 & 5.062 & & \\
\hline Rest pre & 47 & 33.60 & 5.343 & 46 & .001 \\
\hline Rest 6 months & 47 & 3.51 & 5.842 & & \\
\hline Rest pre & 44 & 32.77 & 4.931 & 43 & $<.001$ \\
\hline
\end{tabular}




\begin{tabular}{|c|c|c|c|c|c|}
\hline Rest 12 months & 44 & 29.57 & 5.009 & & \\
\hline Rest post & 49 & 32.06 & 4.789 & 48 & .073 \\
\hline Rest 6 months & 49 & 3.88 & 5.974 & & \\
\hline Rest post & 46 & 31.76 & 4.766 & 45 & .018 \\
\hline Rest 12 months & 46 & 29.67 & 5.051 & & \\
\hline Rest 6 months & 29 & 3.24 & 5.938 & 28 & .752 \\
\hline Rest 12 months & 29 & 29.86 & 5.174 & & \\
\hline SA45GSI pre & 98 & 59.20 & 9.584 & 97 & $<.001$ \\
\hline SA45GSI post & 98 & 56.17 & 8.595 & & \\
\hline SA45GSI pre & 48 & 58.46 & 11.396 & 47 & .001 \\
\hline SA45GSI 6 months & 48 & 53.50 & 9.785 & & \\
\hline SA45GSI pre & 46 & 59.28 & 9.704 & 45 & $<.001$ \\
\hline SA45GSI 12 months & 46 & 54.00 & 9.659 & & \\
\hline SA45GSI post & 48 & 54.88 & 9.141 & 47 & .375 \\
\hline SA45GSI 6 months & 48 & 53.79 & 1.242 & & \\
\hline SA45GSI post & 46 & 56.59 & 7.963 & 45 & .086 \\
\hline SA45GSI 12 months & 46 & 54.13 & 9.863 & & \\
\hline SA45GSI 6 months & 28 & 54.21 & 1.546 & 27 & .291 \\
\hline SA45GSI 12 months & 28 & 52.50 & 1.885 & & \\
\hline SA45PST pre & 98 & 58.79 & 9.454 & 97 & .001 \\
\hline SA45 PST post & 98 & 56.19 & 9.055 & & \\
\hline SA45 PST pre & 48 & 57.25 & 1.850 & 47 & .248 \\
\hline SA45 PST 6 months & 48 & 55.23 & 11.030 & & \\
\hline SA45 PST pre & 46 & 58.61 & 9.441 & 45 & .006 \\
\hline SA45 PST 12 months & 46 & 54.22 & 1.579 & & \\
\hline SA45 PST post & 48 & 54.10 & 9.340 & 47 & .379 \\
\hline SA45 PST 6 months & 48 & 55.35 & 11.154 & & \\
\hline SA45 PST post & 46 & 56.33 & 8.404 & 45 & .124 \\
\hline SA45 PST 12 months & 46 & 54.28 & 1.668 & & \\
\hline SA45 PST 6 months & 28 & 56.43 & 11.279 & 27 & .024 \\
\hline SA45 PST 12 months & 28 & 52.14 & 1.896 & & \\
\hline
\end{tabular}

Note . BMI $=$ Body Mass Index, FCI $=$ Food Craving Inventory, POF $=$ Power of Food Scale, Restraint $=$ Revised Restraint Scale, SA45GSI = Symptom Assessment Global Severity Index, SA45PST = Symptom Assessment Positive Symptom Index.

$(M=58.46, S D=11.39)$ to 6 -month follow-up $(M=53.50, S D=9.78)$ and from pretreatment to 12 -month follow-up $(M=54.00, S D=9.66)$. The total number of self-reported symptoms was significantly lower from pretreatment $(M=58.61$, $S D=9.44)$ to 12 -month follow-up $(M=54.22, S D$ $=10.57)$ and also between 6-month $(M=56.43$, $S D=11.27)$ and 12-month follow-up $(M=52.14$, $S D=1.89$ ).

Table 3 also presents the nonsignificant analysis. The majority of these nonsignificant results highlight little change between the 6- and 12-month follow-up points.

\section{Discussion}

This study examined the effects of EFT for food cravings within the context of a randomized clinical trial. The EFT treatment was offered to overweight and obese participants and was evaluated against a WL condition. It was anticipated that treatment gains reported in Stapleton et al. 
(2011) would be maintained for the EFT group. The results are relatively supportive of the study predictions. This trial confirms other EFT research that has suggested that the techniques effects are enduring (e.g., Rowe, 2005; Wells et al., 2003); this particular follow-up study provides some evidence for the durability of EFT for participants who applied it to their food cravings.

Results at the 6-month follow-up point (Stapleton et al., 2011) indicated that although there were no significant mean differences for weight and BMI, food craving, subjective power over food and restraint remained significantly changed from pretreatment and significant decreases in psychological distress during the treatment phase remained the same at the 6-month point. Post hoc analysis indicated at 12-months the same significant reductions remained stable with the addition of a significant difference from pretreatment for weight and BMI. Although the individual paired sample $t$ tests for the SA45PST (which measured the total number of symptoms reported to be present) did indicate a significant decrease in scores from pre- to posttreatment, pretreatment to 6 months, and pretreatment to 12 months, it did not result in a significant decrease from pretreatment to 12-month follow-up using post hoc analysis. However, it is important to note that the pretreatment to 12-month follow-up result for the SA45PST was significant prior to the Bonferroni correction. The Cohen's $d$ was .67 , which suggests medium to large effect; therefore, the difference from posttreatment to pretreatment could be considered inconclusive, as the power was low.

Most noteworthy is the fact that from posttreatment to 12-month follow-up, there were significant reductions for participants' weight and BMI, which was not significant pre- to posttreatment or at the 6-month follow-up point. The fact that participants did not indicate a significant change in weight or BMI from pre- to posttreatment was expected because of the short (4 week) timeframe of the program. It was hypothesised that by 6 -month follow-up there may have been a significant change in weight and BMI; however, this was not achieved.

There were no significant differences for FCI total scores, POF total score, SA45GSI, and SA45PST scores from posttreatment to 12-month follow-up using post hoc analysis (restraint indicated a decrease from posttreatment to 12-month follow-up in the paired sample $t$ tests). The signifi- cant reductions in these measures achieved from pre- to posttreatment were maintained, however, at the 6-month point (with the exception of restraint, which reached significance at 6 months). The paired $t$-test comparisons across all time points indicated that the SA45PST (total number of psychiatric symptoms reported to be present) at 12-month follow-up was significantly lower than it was at 6 months and when pretreatment was compared to 12-month follow-up. This, coupled with the decrease in global severity scores from pretreatment to 6-month follw-up and pretreatment to 12-month follow-up, appears to signify that the differences achieved over the 4-week program (scores were also significantly lower pre- to posttreatment for both) may have at least stayed stable.

The impact of using EFT appeared to decrease food cravings, and perhaps associated reduced consumption of those foods (and improved food choices) may have occurred. Previous clinical and nonclinical research has indicated that food cravings frequently lead to consumption of the craved food and may be correlated to body weight (Franken \& Muris, 2005; Hill \& HeatonBrown, 1994). Cravings for high fat items have been linked to elevated BMIs (Drewnowski et al., 1985). The results of this study signify that there may be a link between food cravings and subsequent weight, and the impact of reducing cravings in order to reduce weight is therefore of importance to clinicians treating obesity.

Research since the publication of our 6-month study has shed light on how a technique such as EFT, which uses a combination of physical manipulation of acupressure points and cognitive instruction (e.g., verbal statements), may have an impact on any intense emotion or behavior. Harvard Medical School neuroimaging studies have shown that stimulating acupressure points decreases arousal in the amygdala (Feinstein, 2010). It is hypothesized that the amygdala is activated by negative emotion or particularly emotional stimuli, and it continues to respond to learned reactions or initial events (Zald, 2003). In food (as well as drug and alcohol) cravings, the amygdala is activated by food cues (Arana et al., 2003), especially to the intensity of taste (Small et al., 2003) or smell (Anderson et al., 2003). Stimulation of acupressure points (especially while exposed to the craved food, such as was done in our study) may deactivate the amygdala's signals, switching off an alarm indicator. Further repetition 
of the technique continues to decrease the former response of wanting to consume the food and the neural pathways may be altered forever (Feinstein, 2010). Ruden (2010) examined the conditioned fear pathways in his recent neurobiological review and concluded that glutamate receptors, which are present in long-standing signal transmissions, are able to be altered with acupressure approaches. This means the conditioned pathways are permanently eliminated. This explains how EFT can be efficacious for so many different physiological and psychological problems.

Homework was prescribed during the trial, to the effect of simply practicing the technique in between sessions. We could not control what the participants would use the technique for; however, in the 12-month follow-up questionnaire, we queried whether they had used the technique for "other" concerns. Although it had not been directly suggested to them in the trial, participants indicated they used the same technique for concerns such as stress and pain and strong emotions such as anger. While this clinical observation is interesting, it is still important to note that this information came only from those who responded, and the nonresponders may not have used the technique in similar ways or at all since treatment ended. Those who did respond and completed the 6- and 12-month follow-up also revealed that they had "forgotten" the food they had originally targeted in the trial. They indicated this was possibly because it was no longer prominent in their diet, and their cravings for the original food were less than a four out of 10 on a subjective 10-point Subjective Units of Distress scale. The aforementioned description of the role of the amygdala and altering of neural pathways may be further illustrated through our participants' comments.

Strengths of this first clinical trial examining EFT and food cravings include the random allocation of participants to conditions, a waitlist comparison, single clinician delivery of the program, and the use of highly reliable and valid measures of assessment and change. However, future research should aim to include a larger sample, a nontreatment control group, individual versus group treatment comparison, and comparison to gold standard psychological treatments such as cognitive behavioral therapy. Analysis in the present study was not adjusted for any covariates, and despite meticulous procedures, there was missing data at the 12-month follow-up point. This is a limitation in that it is unclear whether nonresponders experienced any improvement as a result of the treatment. Because results such as those reported here may be attributed to a placebo effect, therapist expectancy or perceived pressure from participants to report improvements, or the novelty effect of the treatment, it would be of great interest and benefit to examine the comparison EFT with sham points to ascertain whether EFT works as per the proposed energy meridian theory and neurobiological models.

This study is the first to present 12-month results for EFT and food cravings and has provided evidence for the worth in pursuing further rigorous research in the area. Further replication studies demonstrating the efficacy of emerging strategies such as EFT are warranted and will assist in creating a strong evidence base to offer alternative therapy choices to consumers. Such strategies will not replace clinical approaches to complex concerns such as obesity, but they may offer critical adjunct tools in its management.

\section{References}

Anderson, A. K., Christoff, K., Stappen, I., Panitz, D., Ghahremani, D. G., Glover, G., . . . Sobel, N. (2003). Dissociated neural representations of intensity and valence in human olfaction. Nature Neuroscience, 6, 196-202. http://dx.doi. org/10.1038/nn1001

Arana, F. S., Parkinson, J. A., Hinton, E., Holland, A. J., Owen, A. M., \& Roberts, A. C. (2003). Dissociable contributions of the human amygdale and orbitofrontal cortex to incentive motivation and goal selection. Journal of Neuroscience, 23,9632-9638.

Church, D., \& Brooks, A. J. (2010). The effect of a brief EFT (Emotional Freedom Techniques) self-intervention on anxiety, depression, pain and cravings in healthcare workers. Integrative Medicine: A Clinician's Journal, 10(4), 40-44.

Craig, G. (2008). The EFT manual. Santa Rosa, CA: Energy Psychology Press.

Craig, G., \& Fowlie, A. (1995). Emotional freedom techniques: The manual. Sea Ranch, CA: Author.

Delahanty, L. M., Meigs, J. B., Hayden, D., Williamson, D. A., \& Nathan, D. M. (2002). Psychological and behavioural correlates of baseline BMI in the diabetes prevention program. Diabetes Care, 25, 1992-1998. http://dx.doi.org/10.2337/ diacare.25.11.1992

Drewnowski, A. J., Brunzell, J. D., Sande, K., Iverius, P. H., \& Greenwood, M. R. C. (1985). Sweet tooth reconsidered: Taste responsiveness in human obesity. Physiology and Behaviour, 35, 617-622. http://dx.doi.org/10.1016/00319384(85)90150-7

Elder, C., Ritenbaugh, C., Mist, S., Aickin, M., Schneider, J., Zwickey, H., \& Elmer, P. (2007). Randomized trial of two mind-body interventions for weight-loss maintenance. Journal of Alternative and Complementary Medicine, 13(1), 67-78. http://dx.doi.org/10.1089/acm.2006.6237 
Feinstein, D. (2008). Energy psychology: A review of the preliminary evidence. Psychotherapy: Theory, Research, Practice, Training, 45, 199-213. http://dx.doi.org/10.1037/00333204.45.2.199

Feinstein, D. (2010, November-December). The case for energy psychology. Psychotherapy Networker. Retrieved from http://www.psychotherapynetworker.org/magazine/ currentissue/1155-the-case-for-energy-psychology

Flint, G. A., Lammers, W., \& Mitnick, D. G. (2005) Emotional Freedom Techniques. Journal of Aggression, Maltreatment \& Trauma, 12(1), 125-150. http://dx.doi.org/10.1300/ J146v12n01 07

Franken, I. H. A., \& Muris, P. (2005). Individual differences in reward sensitivity are related to food craving and relative body weight in healthy women. Appetite, 45, 198-201. http://dx.doi.org/10.1016/j.appet.2005.04.004

Garrow, J., \& Summerbell, C. (2000). Obesity. In A. Stevens, J. Raftery, \& J. Mant (Eds.), Health care needs assessment: The epidemiologically based needs assessment reviews (3rd series). Abingdon, Oxfordshire, United Kingdom: Radcliffe Medical Press.

Heatherton, T. F., Herman, C. P., Polivy, J., King, G. A., \& McGree, S. T. (1988). The (mis)measurement of restraint: An analysis of conceptual and psychometric issues. Journal of Abnormal Psychology, 97, 19-28. http://dx.doi. org/10.1037/0021-843X.97.1.19

Herman, C. P., \& Polivy, J. (1980). Restrained eating. In A. J. Standard (Ed.), Obesity (pp. 208-225). Philadelphia, PA: W. B. Saunders.

Hill, A. J., \& Heaton-Brown, L. (1994). The experience of food craving: A prospective investigation in healthy women. Journal of Psychosomatic Research, 38, 801-814. http:// dx.doi.org/10.1016/0022-3999(94)90068-X

Lowe, M. R., Butryn, M. L., Didie, E. R., Annunziato, R. A., Thomas, J. G., Crerand, C. E., . . . Halford, J. (2009). The Power of Food Scale: A new measure of the psychological influence of the food environment. Appetite, 53, 114-118. http://dx.doi.org/10.1016/j.appet.2009.05.016

Karatzias, T., Power, K., Brown, K., McGoldrick, T., Begum, M., Young, J., . . . Adams, S. (2011). A controlled comparison of the effectiveness and efficiency of two psychological therapies for Posttraumatic Stress Disorder, Eye Movement Desensitization and Reprocessing vs. Emotional Freedom Techniques. Journal of Nervous and Mental Disorders, 199, 372-378. http://dx.doi.org/10.1097/ NMD.0b013e31821cd262

Kemps, E., \& Tiggemann, M. (2010). A cognitive experimental approach to understanding and reducing food cravings. Current Directions in Psychological Science, 19(2), 86-90. http://dx.doi.org/10.1177/0963721410364494

Rowe, J. (2005). The effects of EFT on long-term psychological symptoms. Counselling and Clinical Psychology Journal, 2(3), 104.
Ruden, R. (2010). When the past is always present: Emotional traumatization, causes, and cures. New York, NY: Routledge.

Ruderman, A. J., \& Besbeas, M. (1992). Psychological characteristics of dieters and bulimics. Journal of Abnormal Psychology, 101, 383-390. http://dx.doi.org/10.1037/0021843X.101.3.383

Salas, M., Brooks, A., \& Rowe, J. (2011). The immediate effect of a brief energy psychology intervention (Emotional Freedom Techniques) on specific phobias: A pilot study. Explore, 7, 155-161. http://dx.doi.org/10.1016/j.explore.2011.02.005 Sandín, B., Valiente, R., Chorot, P., Santed, M., \& Lostao, L. (2008). SA-45: A brief form of the SCL-90. Psicothema, 20, 290-296.

Shaw, K., O’Rourke, P., Del Mar, C., \& Kenardy, J. (2005). Psychological interventions for overweight or obesity. Cochrane Database of Systematic Reviews, 2005(2). http:// dx.doi.org/10.1002/14651858.CD003818.pub2

Small, D. M., Gregory, M. D., Mak, Y. E., Gitelman, D. R., Mesulam, M., \& Parrish, T. B., (2003). Dissociation of neural representation of intensity and affective valuation in human gustation. Neuron, 39, 701-711.

SPSS for Windows (Version 17.0.0) [computer program]. (2008). Chicago, IL: SPSS.

Stapleton, P. B., Sheldon, T., Porter, B., \& Whitty, J. (2011). A randomised clinical trial of a meridian-based intervention for food cravings with six month follow-up. Behaviour Change, 28(1), 1-16. http://dx.doi.org/10.1375/bech.28.1.1

Stein, P., \& Brooks, A. (2011). Efficacy of EFT provided by coaches versus licensed therapists in veterans with PTSD. Energy Psychology Journal: Theory, Research, \& Treatment, 3(1), 33-42.

Strategic Advantages. (2000). Symptom Assessment-45 Questionnaire (SA-45). New York, NY: Multi-Health Systems.

Weingarten, H. P., \& Elston, C. (1990). The phenomenology of food cravings. Appetite, 15, 231-246. http://dx.doi. org/10.1016/0195-6663(90)90023-2

Wells, S., Polglase, K., Andrews, H., Carrington, P., \& Baker, A. H. (2003). Evaluation of a meridian-based intervention, Emotional Freedom Techniques (EFT), for reducing specific phobias of small animals. Journal of Clinical Psychology, 59, 943-966. http://dx.doi.org/10.1002/jclp.10189

White, M. A., Whisenhunht, B. L., Williamson, D. A., Greenway, F. L., \& Netemeyer, R. G. (2002). Development and validation of the Food-Craving Inventory. Obesity Research, 10, 107-114. http://dx.doi.org/10.1038/oby.2002.17

Withrow, D., \& Alter, D. A. (2010). The economic burden of obesity worldwide: A systematic review of the direct costs of obesity. Obesity Reviews, 12, 131-141. doi: 10.1111/j.1467789X.2009.00712.x.

Zald, D. H. (2003). The human amygdala and the emotional evaluation of sensory stimuli. Brain Research Reviews, 41, 88-123. http://dx.doi.org/10.1016/S0165-0173(02)00248-5 